\title{
Chromosomally-mediated antibiotic resistance and virulence
}

Although the relationship between plasmid-mediated antibiotic resistance and alterations in virulence is established, ${ }^{1-4}$ the association of chromosomallymediated resistance and virulence has received less attention. Spontaneous mutations in chromosomal genes occur at a frequency of $10^{6}-10^{8} /$ cell division. In relation to antibiotic resistance, such mutations usually involve genes encoding the target site, or cell structures affecting access to the target site. In practice, chromosomal mutations are found less frequently in natural bacterial populations probably because they reduce the fitness of bacteria to survive. In the field of medical microbiology this may result from the loss or alteration of a virulence factor. ${ }^{5-6}$

Chromosomal mutations conferring antibiotic resistance via alterations in outer-membrane porins of gram-negative bacteria may affect virulence. Porins allow the transport across bacterial membranes of nutrients, ions and certain antibiotics. A complex control mechanism regulates the quantitative production of porins and affects the sensitivity of the bacterial cell to various antimicrobial agents such as the quinolones, $\beta$-lactam antibiotics and tetracyclines. The presence of porins of different sizes will affect the range of substances entering the cell. Mutations that affect the transcriptional regulator that controls the ratio of different porins, Omp $\mathbf{R}$, can result in strains with markedly reduced virulence. ${ }^{?}$

Cyclic AMP (cAMP) and the cAMP receptor protein are essential for the transcription of many genes and operons concerned with the transport and breakdown of catabolites. The cAMP concentration in cells also influences the synthesis of pili, fimbriae, flagella and at least one outer-membrane protein; all these factors are related to pathogenicity. ${ }^{8}$ Deficiency in cAMP and cAMP receptor protein can also be associated with resistance to $\beta$-lactam antibiotics. Curtiss and Kelly ${ }^{8}$ developed strains of Salmonella typhimurium that were unable to synthesise adenyl cyclase and cAMP receptor protein and thus were deficient in cAMP. These mutant strains required significantly higher infective doses than the parent strain in a mouse model.

In studies of the relationship between antibiotic resistance and pathogenicity the direct comparison of sensitive and resistant bacterial strains in virulence assays has been a favoured approach. In one study, aminoglycoside resistant mutants of Pseudomonas aeruginos $a$ were examined for alterations in virulence. ${ }^{9}$ The mechanism of aminoglycoside resistance was either a decrease in cytochrome and nitrate reductase activity or the presence of uncapped subunits of lipopolysaccharide (LPS). The possession of smooth LPS is an established virulence factor in gram-negative bacteria. All mutant strains were less virulent in a mouse model and the strains with altered cytochrome activity also demonstrated slower growth rates in vitro. ${ }^{9}$ In a similar study, 4-quinolone-resistant $P$. aeruginosa obtained by serial culture through subinhibitory concentrations of the quinolones, exhibited decreased virulence for mice and also increased sensitivity to aminoglycosides. ${ }^{10}$ Escherichia coli mutants resistant to the 4-quinolones failed to haemagglutinate because of a lack of surface pili. ${ }^{11}$ It was suggested that these 4-quinolone-resistant $E$. coli may be less capable of colonising the urinary tract and initiating infection. ${ }^{11}$

Chromosomally-mediated resistance of Staphylococcus aureus to several antibiotics has been shown to be associated with altered virulence. As early as 1944 , penicillin-resistant $S$. aureus strains were shown to have reduced pathogenicity in mice and reduced production of coagulase and protein A compared to sensitive strains. ${ }^{12,13}$

Exposure of $S$. aureus to increasing concentrations of gentamicin often results in bacteria that grow as small non-haemolytic colonies and do not produce coagulase or DNAase or ferment mannitol. ${ }^{14}$ Some methicillin-resistant $S$. aureus produce more coagulase but less protein A than methicillin-sensitive strains ${ }^{15,16}$ and are more likely to produce $\alpha$-haemolysin and enterotoxin A. ${ }^{17,18}$ Rifampicin-resistant mutants of $S$. aureus exhibit altered exoprotein production and are less virulent for mice. ${ }^{19,20}$ Some strains of $S$. aureus that are resistant to ofloxacin appear to lose the ability to produce coagulase.$^{21}$ However, in a more extensive study, ciprofloxacin- resistant and ciprofloxacin-sensitive strains of $S$. aureus produced similar amounts of coagulase, protein $\mathrm{A}, \alpha$-haemolysin and $\delta$-haemolysin, grew at a similar rate and were equally virulent in a subcutaneous abscess model in mice (unpublished observation).

Bacteria exhibiting changes associated with chromosomal mutations may show a reduction in pathogenicity and infectivity. Further studies examining the pathogenicity of antibiotic-resistant mutants will help assess their potential both to initiate infection and to sustain infection, if the resistance-conferring mutations arise during therapy.

S. A. Doss

Department of Medical Microbiology, Medical School, University of Edinburgh, Teviot Place, Edinburgh EH8 9AG. 


\section{References}

1. Laporta MZ, Silva MLM, Scaletsky ICA, Trabulsi LR. Plasmids coding for drug resistance and localized adherence to HeLa cells in enteropathogenic Escherichia coli O55:H and O55:H6. Infect Immun 1986; 51: 715-717.

2. Wachsmuth IK, Falkow S, Ryder RW. Plasmid-mediated properties of heat-stable enterotoxin-producing Escherichia coli associated with infantile diarrhea. Infect Immun 1976; 14: 403-407.

3. Echeverria P, Verhaert L, Ulyangco CV et al. Antimicrobial resistance and enterotoxin production among isolates of Escherichia coli in the Far East. Lancet 1978; 2: 589-592.

4. Lacey RW, Chopra I. Effect of plasmid carriage on the virulence of Staphylococcus aureus. J Med Microbiol 1975; 8: 137-147.

5. Smith JT. Mechanisms of drug resistance. In: Edwards DI, Hiscock DR (eds) Chemotherapeutic strategy. 1983: 79-83.

6. Saunders JR. Genetics and evolution of antibiotic resistance. $\mathrm{Br}$ Med Bull 1984; 40: 54-60.

7. Dorman CJ, Chatfield S, Higgins CF, Hayward C, Dougan G. Characterisation of porin and $O m p R$ mutants of a virulent strain of Salmonella typhimurium: Omp $R$ mutants are attenuated in vivo. Infect Immun 1989; 57: 2136-2140.

8. Curtiss R, Kelly SM. Salmonella typhimurium deletion mutants lacking adenylate cyclase and cyclic AMP receptor protein are avirulent and immunogenic. Infect Immun 1987; 55: 3035-3043.

9. Bryan LE, Godfrey AJ, Schollardt T. Virulence of Pseudomonas aeruginosa strains with mechanisms of microbial persistence for $\beta$-lactam and aminoglycoside antibiotics in a mouse infection model. Can J Microbiol 1985; 31:377-380.

10. Ravizzola G, Pirali F, Paolucci A et al. Reduced virulence in ciprofloxacin-resistant variants of Pseudomonas aeruginosa strains. J Antimicrob Chemother 1987; 20: 825-829.

11. Crumplin GC. Gram negative mutants resistant to 4-quino- lones: are they competent pathogens? In: Crumplin GC (ed) The 4-quinolones: antibacterial agents in vitro. London, Springer Verlag. 1990: 257-268.

12. Rake G, McKee CM. Hamre DM, Houck CL. Studies on penicillin II. Observations on therapeutic activity and toxicity. J Immunol 1944; 48: 271-289.

13. Blair JE, Carr M, Buchman J. Action of penicillin staphylococci. J Immunol 1946; 52: 281-292.

14. Musher DM, Baughn RE, Templeton GB, Minuth JN. Emergence of variant forms of Staphylococcus aureus after exposure to gentamicin and infectivity of variants in experimental animals. J Infect Dis 1977; 136: 360-369.

15. Lacey RW, Barr KW, Barr VE, Inglis TJ. Properties of methicillin-resistant Staphylococcus aureus colonizing patients in a burns unit. $J$ Hosp Infect 1986; 7: 137-148.

16. Roberts JI, Gaston MA. Protein A and coagulase expression in epidemic and non-epidemic Staphylococcus aureus. J Clin Pathol 1987; 40: 837-840.

17. Jordens JZ, Duckworth GJ, Williams RJ. Production of "virulence factors" by "epidemic" methicillin-resistant Staphylococcus aureus in vitro. J Med Microbiol 1989; 30 : 245-252.

18. Coia JE, Browning L, Haines L, Birkbeck TH, Platt DJ. Comparison of enterotoxins and haemolysins produced by methicillin-resistant (MRSA) and -sensitive (MSSA) Staphylococcus aureus. J Med Microbiol 1992;36: 164-171.

19. Al-Ani B, Aboshkiwa M, Glass RE, Coleman G. The patterns of extracellular protein formation by spontaneouslyoccurring rifampicin-resistant mutants of Staphylococcus aureus. FEMS Microbiol Lett 1990; 70: 91-94.

20. Moorman DK, Mandell GL. Characteristics of rifampicinresistant variants obtained from clinical isolates of Staphylococcus aureus. Antimicrob Agents Chemother 1981; 20: 709-713.

21. Smith JT. In vitro and in vivo mutation frequencies to resistance-do they correlate in the long term? In: Crumplin GC (ed) The 4-quinolones: antibacterial agents in vitro. London, Springer Verlag. 1990: 215-228. 